\title{
BMJ Open Does the index-to-ring finger length ratio (2D:4D) differ in amyotrophic lateral sclerosis (ALS)? Results from an international online case-control study
}

Jane Alana Parkin Kullmann, Roger Pamphlett

To cite: Parkin Kullmann JA, Pamphlett R. Does the indexto-ring finger length ratio (2D:4D) differ in amyotrophic lateral sclerosis (ALS)? Results from an international online case-control study. BMJ Open 2017;7:e016924. doi:10.1136/ bmjopen-2017-016924

- Prepublication history and additional material are available. To view these files please visit the journal online (http://dx.doi. org/10.1136/bmjopen-2017016924)

Received 21 March 2017 Revised 7 June 2017 Accepted 16 June 2017

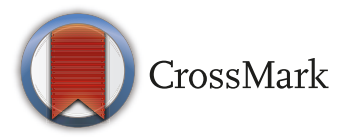

Discipline of Pathology, Sydney Medical School, Brain and Mind Centre, The University of Sydney, Sydney, Australia

Correspondence to Professor Roger Pamphlett; roger.pamphlett@sydney.edu.au

\section{ABSTRACT}

Objectives The ratio of the length of the index finger (2D) to the ring finger (4D) (2D:4D) has been reported to be lower (ie, 2D<4D) in people with amyotrophic lateral sclerosis (ALS) than non-ALS controls. This has led to suggestions that exposure to increased prenatal testosterone, which also lowers this ratio, could be a risk factor for ALS. In an attempt to test this hypothesis, we examined 2D:4Ds from large numbers of patients with ALS and controls.

Setting An online multilingual questionnaire enabling respondents to measure their own index and ring finger lengths.

Participants of the initial 949 respondents, 572 remained for analysis after elimination for inability to straighten fingers, not answering the question, statistical outliers and aged $<40$ years. Respondents remaining for analysis were 202 patients with ALS (125 males, 77 females) and 370 non-ALS controls (112 males, 258 females).

Results Unpaired t-tests with $95 \% \mathrm{Cls}$ were used to assess differences in mean 2D:4Ds. Males had significantly lower mean 2D:4Ds than females, in both ALS and control groups, for both left and right hands. No significant differences were found in 2D:4Ds between ALS and control groups, in either males or females, for either left or right hands. Receiver operating characteristic curves showed no power for 2D:4Ds to predict ALS status in either males or females.

Conclusions 2D:4Ds did not differ between patients with ALS and controls in this study. This was despite the dataset being large enough to confirm the established finding of lower 2D:4Ds in males compared with females. These findings do not support the hypothesis that exposure to increased prenatal testosterone is a risk factor for ALS. A putative lower 2D:4D has been proposed to explain the link between ALS and exercise, but our results indicate that other exercise-related factors are more likely to explain this association.

\section{INTRODUCTION}

Gender differences in the ratio of the length of the index finger (2D) to the ring finger (4D) (2D:4D) have been studied for over a century, with a lower mean ratio (ie, $2 \mathrm{D}<4 \mathrm{D}$ ) being repeatedly found in males compared

\section{Strengths and limitations of this study}

- Use of an online questionnaire enabled recruitment of larger numbers of patients with amyotrophic lateral sclerosis (ALS) and controls than previously available for this type of study.

- The larger numbers of respondents allowed for statistically robust separate analyses of male and female length of the index finger (2D) to the ring finger (4D) ratios.

- The accuracy of self-measured finger lengths was validated by the finding of similar gender differences ( $2 \mathrm{D}<4 \mathrm{D}$ in males, $2 \mathrm{D} \geq 4 \mathrm{D}$ in females) to those reported in previous studies.

- This was an international study, with comparable national, ethnic and cultural backgrounds in ALS and non-ALS respondents.

- Although patients with ALS had to self-report their disease status, self-reporting of ALS compared with physician's assessments has been shown to be accurate in over $90 \%$ of participants.

- $10 \%$ of ALS patients and $2 \%$ of controls were unable to straighten their fingers to provide measurements.

- Numbers of respondents are too limited to investigate interethnic and internation 2D:4D differences.

- More random errors and larger SD are generally found in self-reported data.

with females, ${ }^{1-3}$ though with considerable overlap. The smaller average male $2 \mathrm{D}: 4 \mathrm{D}$ is considered to be due to increased amounts of intrauterine testosterone relative to oestrogen. ${ }^{45}$ The reason for this appears to be that during development the fetal ring finger has more plentiful androgen and oestrogen receptors than the index finger and so grows longer in the presence of a relative excess of testosterone. ${ }^{67}$

The 2D:4D has been used as an index of prenatal hormone exposure in a variety of physiological and psychological conditions, including athletic ability and strength, ${ }^{3} 8-10$ fertility, ${ }^{4}$ various behaviours ${ }^{11}$ and sexual orientation. ${ }^{12}$ The ratio has also been reported to 
be different in diseases where gender imbalances can be marked or in which prenatal influences are suspected, including breast cancer, ${ }^{13}$ prostate cancer, ${ }^{14}$ Alzheimer's disease $^{15}$ and multiple sclerosis. ${ }^{16}$

Amyotrophic lateral sclerosis (ALS), also known as motor neuron disease (MND), is a progressive neurodegenerative disease with a usual postdiagnosis survival time of 2-5years. ${ }^{17}$ A previous study reported a lower mean 2D:4D in patients with ALS compared with controls. ${ }^{18}$ However, subject numbers in this study were too limited to detect the lower 2D:4D usually reported in males. To see if the results of this study could be validated with a larger number of subjects, we used data from an international online questionnaire ${ }^{19}$ in which ALS and non-ALS respondents self-measured the lengths of their ring and index fingers.

\section{METHODS}

\section{Setting}

This is a case-control study using data from an international, multilingual, web-based questionnaire ('ALS Quest') designed to look for risk factors for ALS. Details on the design and implementation of the questionnaire have been published. ${ }^{19}$ The full questionnaire, which uses Qualtrics survey software, can be viewed at www. alsquest.org and completed online. A PDF version of the questionnaire is available for downloading as online supplementary file $\mathrm{S} 1$.

Responses to the questionnaire collected between January 2015 and February 2017 were used for analysis. Respondents for the questionnaire were recruited via national and state ALS/MND associations, national ALS registries, the internet and social media. No personally identifying data were collected to allow the respondents to remain anonymous. The project was approved by the Human Ethics Committee of the Sydney Local Health District.

Cases were respondents who replied to a question about having been diagnosed as having ALS with 'Yes, I have been diagnosed with ALS/MND'. Controls were participants who answered this question with 'No, I have not been diagnosed with ALS/MND'. To maintain anonymity, information on disease status was not requested from attending physicians but was self-reported.

\section{Finger measurements}

The guide on how to perform finger measurements consisted of a diagram (figure 1) together with the following instructions: 'Please measure the lengths of your index (second) and ring (fourth) fingers on each hand. The diagram below shows you how to make the measurements. If you can only straighten out the fingers on one hand, only record the measurements for that hand. If possible, please take off any rings prior to measuring your fingers. First lay the back of your hand out flat on a level surface, with your fingers together. Then, with a rigid millimetre ruler (not a tape measure), measure

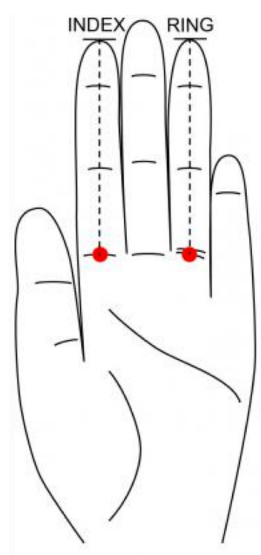

LEFT

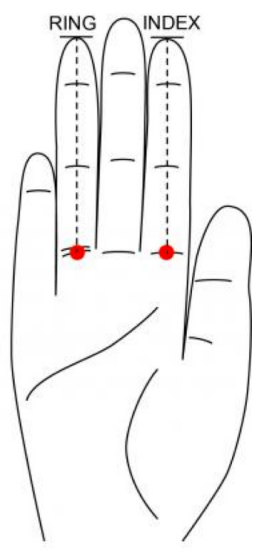

RIGHT
Figure 1 The diagram that appears in the online questionnaire to assist respondents to measure their finger lengths.

from (1) the tip of the finger to (2) the middle of the crease at the bottom of the finger. The ring finger often has two of these creases, so here measure from the crease that is closest to the wrist'. Drop-down lists with numbers ranging from 35 to $135 \mathrm{~mm}$ allowed respondents to select the relevant finger lengths.

\section{Other data analysed}

Other questionnaire data used in the study (answers mostly selected from drop-down lists) were: date when consent was given to conduct the questionnaire, age, gender, country of residence, country of birth, ancestry, cultural group, dominant hand and family history of ALS. People with ALS were asked their date of diagnosis, subtype of ALS and to complete the ALS Functional Rating Scale (ALSFRS-R) ${ }^{20}$ which for this study was scored in reverse so that higher scores indicated more advanced disease.

\section{Statistical analyses}

Data from the survey software were transferred to a Microsoft Excel file, where 2D:4Ds were calculated by dividing the length of the index finger by the length of the ring finger. IBM SPSS Statistics for Macintosh (V.22) and GraphPad Prism V.7 were used to create percentage frequency histograms, calculate unpaired t-tests with 95\% CIs to compare means and perform Pearson's test for correlations. Receiver operating characteristic (ROC) curves assessed the sensitivity and specificity of 2D:4Ds to distinguish between ALS and controls. Effect sizes were calculated using $G^{*}$ Power. $^{21}$ Significance was assessed at the 0.05 level.

\section{Exclusion criteria}

Respondents were excluded from analysis (figure 2) if they: (1) could not straighten the fingers of both their hands, (2) did not answer the finger length question, (3) had extreme outlier results (identified on SPSS as being more than three times the IQR) and (4) were under the age of 40 years (to limit the number of control 


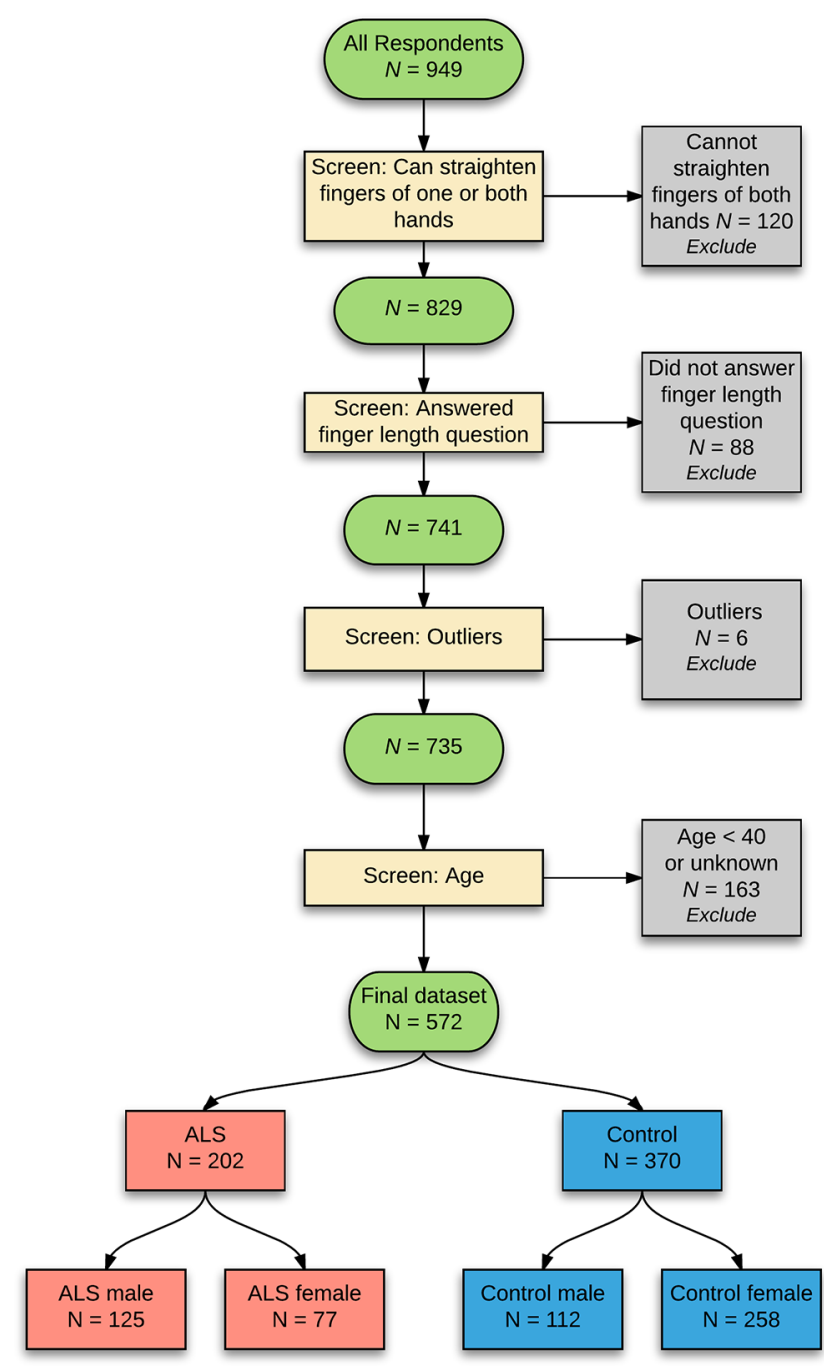

Figure 2 Flow chart of the selection of study respondents. ALS, amyotrophic lateral sclerosis.

respondents who might later be diagnosed with ALS) or did not answer the age question.

\section{RESULTS}

\section{Cases and controls}

Extreme outliers were five 2D:4D results and one left hand/right hand 2D:4D difference; when these were excluded, all self-measured values produced ratios ranging from 0.8 to 1.2 , the range considered to be likely to reflect real values. ${ }^{22}$ After all exclusion criteria were applied, the original number of 949 respondents was reduced to a final dataset number of 572 (figure 2). These remaining respondents comprised 202 ALS cases (125 male, 77 female) and 370 non-ALS controls (112 male, 258 female). The mean age of ALS patients was 61.7 years (SD 9.1 years, range 40-83 years) and of controls was 57.4 years (SD 10.4 years, range $40-86$ years). Original finger length measurements from the analysed respondents (with genders and ages) are available as online supplementary file S2.

\section{Sources of information about the questionnaire}

The most common sources of information about the questionnaire cited by respondents were: ALS associations (36\% of respondents), the internet $(22 \%)$, friends $(9 \%)$, patients with ALS (7\%), health professionals (6\%), community groups (5\%), Facebook $(4 \%)$, the USA CDC National ALS Registry (3\%), the Canadian Neuromuscular Disease Registry (2\%) and ALS researchers (2\%).

\section{Demographic and clinical characteristics}

The composition of the ALS and control groups was similar with regards to country of birth, country of residence, ancestry and cultural group (table 1 ). The majority of respondents resided in Australia, the USA and Canada, though residents of a further 26 countries supplied responses. As reported by the ALS respondents, $52 \%$ had 'classic' (upper and lower motor neuron variant) ALS, $10 \%$ progressive bulbar palsy, $10 \%$ primary lateral sclerosis (upper motor variant), $7 \%$ progressive muscular atrophy (lower motor neuron variant), 9\% 'other' and 9\% did not know their subtype of ALS. Seven per cent of ALS patients had a relative with ALS and were classified as 'familial' ALS. Most controls (68\%) reported no relatives with ALS, whereas $24 \%$ had one relative with ALS and $8 \%$ had more than one relative with ALS.

\section{Comparison of 2D:4Ds between males and females}

Left and right hand 2D:4Ds were significantly smaller in males compared with females, both in patients with ALS and controls (figure 3 ). The mean difference was slightly greater in patients with ALS than in controls (table 2).

\section{Comparison of 2D:4Ds between patients with ALS and controls}

Because of the mean 2D:4D differences found between our male and females, comparisons between ALS and control groups were undertaken in males and females separately. The frequency distributions of 2D:4Ds from both the left and right hands of ALS and control respondents were similar (figure 4). No significant differences in mean 2D:4Ds were seen between ALS and controls groups, in either the left or right hands, of either males or females (figure 3, table 2). Mean 2D:4Ds differed at only the third decimal place between these groups, and the 95\% CIs spanned the zero difference in means (figure 3).

ROC curves of left and right hands showed that 2D:4Ds did not predict ALS status in either males or females (figure 5). The curves were close to the diagonal along the line of no-discrimination, with areas under the curve close to 0.5 , indicating that the 2D:4Ds were neither sensitive nor specific to ALS status.

\section{Rate of ALS progression}

An estimate of the rate of ALS progression was obtained by dividing the reverse ALSFRS-R score by the number of months since diagnosis. The rate of ALS progression did not correlate significantly with 2D:4D, in either the left hand ( $\mathrm{r} 0.072, \mathrm{p} 0.317$ ) or the right hand ( $\mathrm{r} 0.082, \mathrm{p}$ 
Table 1 Demographic characteristics of respondents

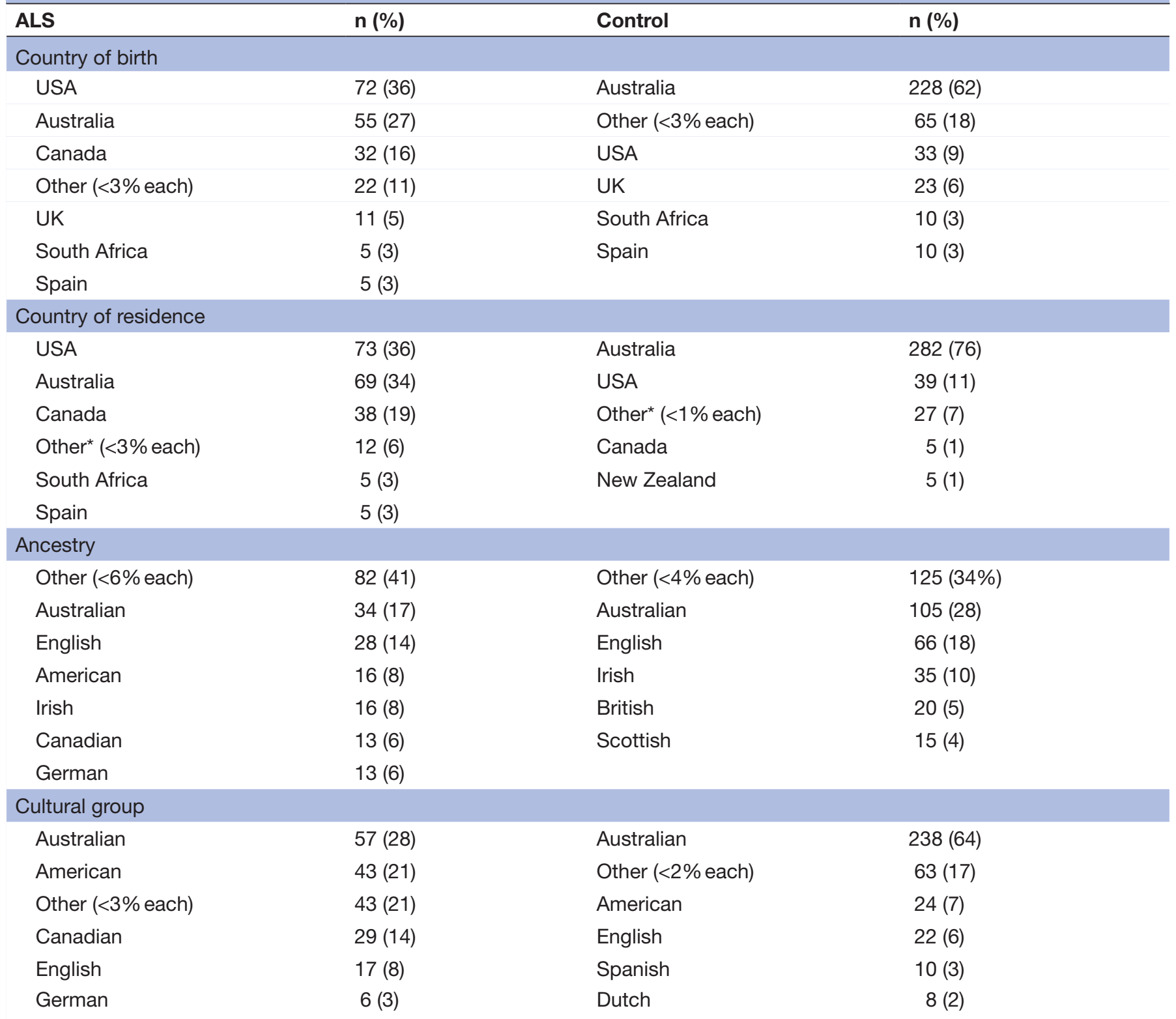

*Other countries of residence (in alphabetical order): Argentina, Belgium, Brazil, China, Colombia, Denmark, Ecuador, Egypt, Finland, Germany, Iran, Ireland, Italy, Japan, Lebanon, Luxembourg, Mexico, the Netherlands, Portugal, Russia, Slovakia, South Korea, Sweden, Switzerland, Turkey and UK.

Some subsets do not add up to the total number of respondents because of answers to particular questions not being given.

ALS, amyotrophic lateral sclerosis.

0.251), indicating the ratio had no influence on disease progression.

\section{Sporadic and familial ALS}

People with sporadic ALS (93\% of cases) had no significant differences in 2D:4Ds to those with familial ALS $(7 \%)$, for either the left or right hands, in either males or females. Similarly, no significant differences were seen in 2D:4Ds between non-ALS controls who had either no family member with ALS (68\%), one family member with ALS $(24 \%)$ or two or more family members with ALS $(8 \%)$, for either hand in either gender.

\section{Left-right symmetry of finger lengths and 2D:4Ds}

No significant differences were found between the lengths of the left and right index fingers, or the left and right ring fingers, in either gender or either disease status group, indicating no asymmetry of left-right finger lengths in our respondents.

No significant differences between left and right hand 2D:4Ds of individual respondents were found, suggesting that respondents did not have difficulty measuring fingers of either their left or right hands. 2D:4Ds were also compared between male/female and ALS/control groups using dominant or non-dominant hands for 


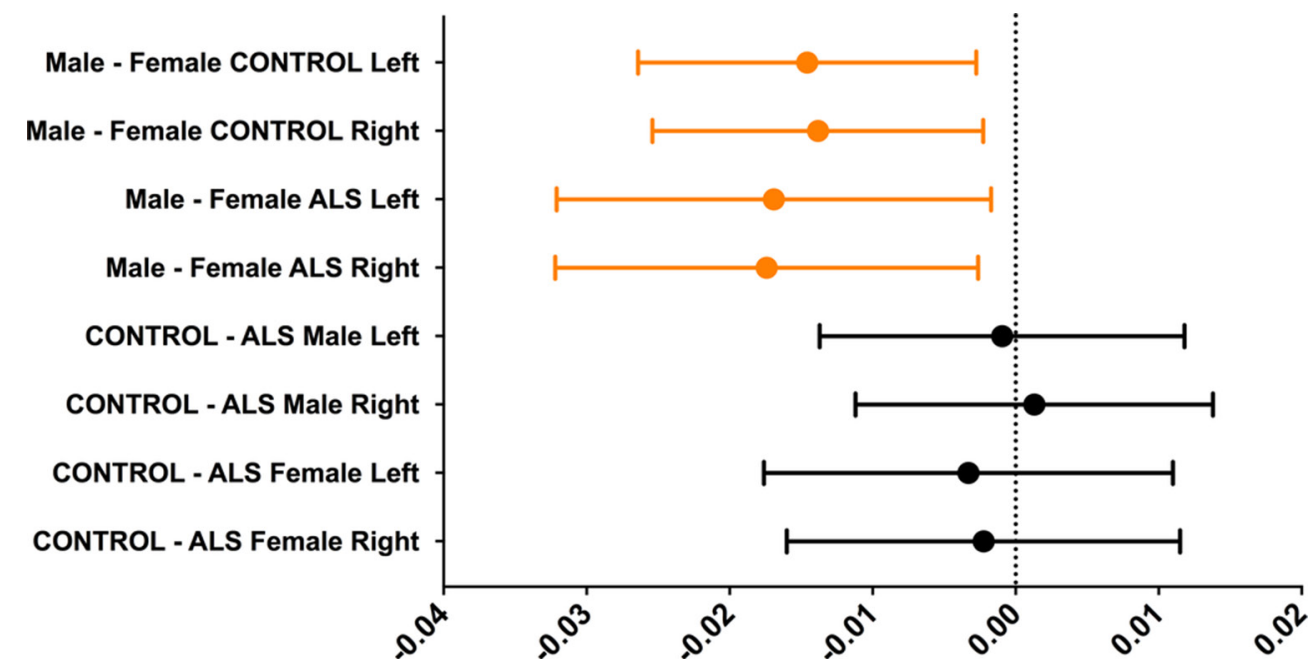

Mean 2D:4D differences (bars: $95 \% \mathrm{Cls}$ )

Figure 3 Differences in mean 2D:4Ds between male and females (upper segment, orange) and between patients with ALS and controls (lower segment, black). Mean 2D:4Ds (filled circles) are significantly smaller in males compared with females, in both control and ALS groups. No differences are seen in mean 2D:4Ds between control and ALS respondents, for either males or females, with the mean differences all close to zero, and the $95 \% \mathrm{Cls}$ (bars) spanning the zero difference (dotted line). 2D:4D, ratio of the length of the index finger (2D) to the ring finger (4D); ALS, amyotrophic lateral sclerosis

measuring $(85 \%$ of respondents were right-hand dominant, $10 \%$ left-hand dominant and $5 \%$ ambidextrous). The results of these analyses were the same as above (ie, males had lower 2D:4Ds, but no 2D:4D differences were found between patients with ALS and controls), indicating that use of the non-dominant hand to hold the ruler did not affect measurements.

Table 2 Differences in mean 2D:4D ratios (with $p$ values and effect sizes) between males and females, and between patients with ALS and controls

\begin{tabular}{|c|c|c|c|c|}
\hline Groups (n) & Mean 2D:4D (SD) & Mean 2D:4D difference $(95 \% \mathrm{Cl})$ & $\mathrm{p}$ Value & Effect size (d) \\
\hline \multicolumn{5}{|l|}{ Male-female left hand } \\
\hline Male control (112) & $0.978(0.0467)$ & $-0.015(-0.026$ to -0.0028$)$ & 0.016 & 0.28 \\
\hline Female control (258) & $0.993(0.0557)$ & & & \\
\hline Male ALS (124) & $0.979(0.0521)$ & $-0.017(-0.032$ to -0.0017$)$ & 0.029 & 0.32 \\
\hline Female ALS (75) & $0.996(0.0537)$ & & & \\
\hline \multicolumn{5}{|l|}{ Male-female right hand } \\
\hline Male control (111) & $0.979(0.0461)$ & $-0.014(-0.025$ to -0.0023$)$ & 0.019 & 0.28 \\
\hline Female control (258) & $0.993(0.0542)$ & & & \\
\hline Male ALS (124) & $0.978(0.0508)$ & $-0.017(-0.032$ to -0.0026$)$ & 0.021 & 0.34 \\
\hline Female ALS (77) & $0.995(0.0529)$ & & & \\
\hline \multicolumn{5}{|l|}{ Control-ALS left hand } \\
\hline Male control (112) & $0.978(0.047)$ & $-0.00094(-0.014$ to 0.012$)$ & 0.884 & \\
\hline Male ALS (124) & $0.979(0.0521)$ & & & \\
\hline Female control (258) & $0.993(0.0557)$ & $-0.0033(-0.018$ to 0.011$)$ & 0.649 & \\
\hline Female ALS (75) & $0.996(0.0537)$ & & & \\
\hline \multicolumn{5}{|l|}{ Control-ALS right hand } \\
\hline Male control (111) & $0.979(0.0461)$ & $0.0013(-0.011$ to 0.014$)$ & 0.835 & \\
\hline Male ALS (124) & $0.978(0.0508)$ & & & \\
\hline Female control (258) & $0.993(0.0542)$ & $-0.0022(-0.016$ to 0.012$)$ & 0.751 & \\
\hline Female ALS (77) & $0.995(0.0529)$ & & & \\
\hline
\end{tabular}

$2 D: 4 D$, ratio of the length of the index finger (2D) to the ring finger (4D); ALS, amyotrophic lateral sclerosis. 

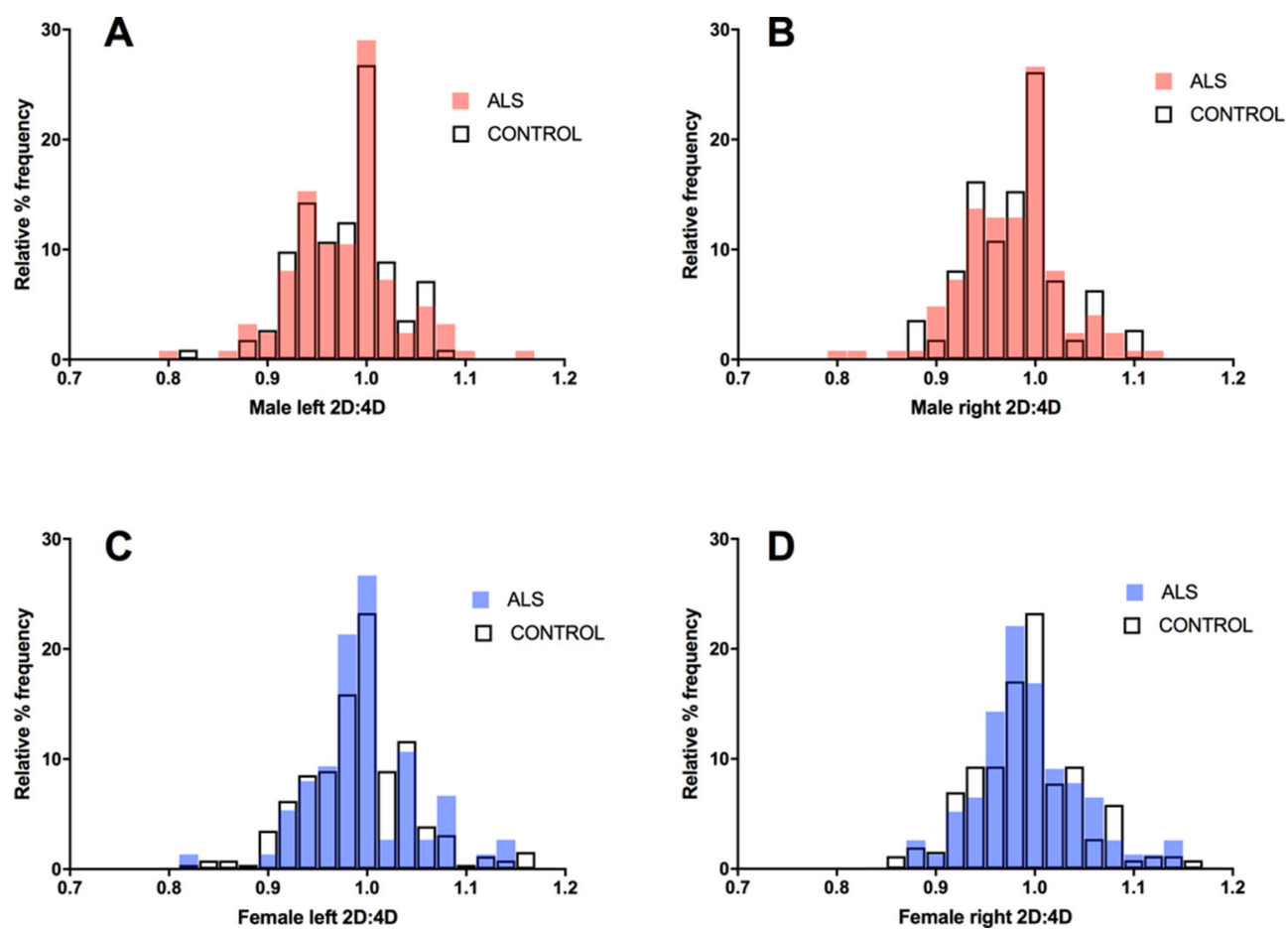

Figure 4 Overlapping histograms show the similar percentage frequency distributions of 2D:4Ds between ALS and control respondents, for both males (A: left hand, B: right hand) and females (C: left hand, D: right hand). 2D:4D, ratio of the length of the index finger (2D) to the ring finger (4D); ALS, amyotrophic lateral sclerosis.

\section{DISCUSSION}

We found no differences in mean 2D:4Ds between ALS and non-ALS respondents, with males and females being analysed separately, and 2D:4Ds had no ability to predict ALS status. This was despite our dataset being large enough to confirm the established lower mean 2D:4D found in males compared with females.

Internet surveys are able to gather data from large numbers of participants, but concerns have been raised about superficial answering of questions and multiple responses from individuals. ${ }^{23}$ These would be unlikely in the present survey, with most respondents either having the serious condition of ALS or having knowledge about the disease. Furthermore, the ALS Quest survey is detailed
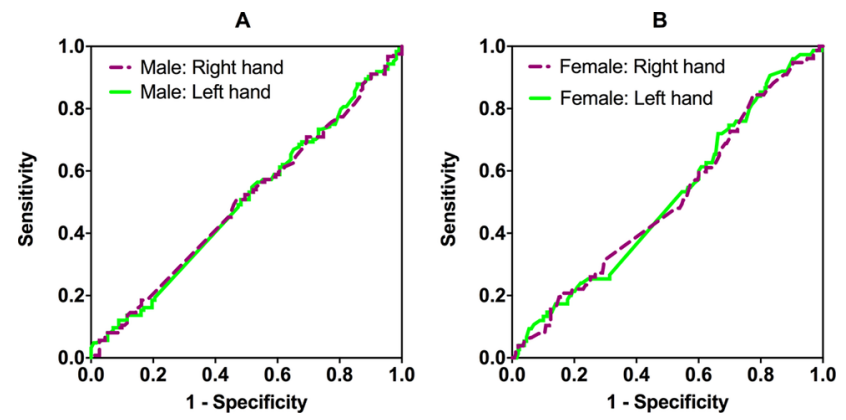

Figure 5 Receiver operating characteristic curves of 2D:4Ds in both males (A) and females (B) are close to the diagonal line of no-discrimination (not drawn since it would obscure the curves). Areas under the curve are close to 0.5 , indicating that the 2D:4Ds are neither sensitive nor specific to ALS status. 2D:4D, ratio of the length of the index finger (2D) to the ring finger (4D); ALS, amyotrophic lateral sclerosis. and extensive, taking most respondents about 2 hours to complete, often in multiple sessions, so respondents who complete the survey are likely to be motivated to give accurate data. Multiple answers are also not likely since the questionnaire is set up to allow only one response per internet browser/computer.

An important issue concerns the accuracy of the self-measured finger lengths in this study. Comparisons between large numbers of Internet and conventionally collected survey data have shown consistency with researcher-measured samples. $^{23}$ Web-based 2D:4D measurements appear to add some random noise to the data, but do not lead to systemic errors. ${ }^{24}$ When self-reported finger measurements were compared with those measured on photocopies by researchers, the self-reported lengths were found to be valid for the study of 2D:4D, provided outliers were removed and large sample numbers were available. ${ }^{25}$ These recommendations were followed in the present study: after a few extreme outliers (only $1 \%$ of the total) were removed, our self-reported 2D:4Ds produced ratios ranging from 0.8 to 1.2. Values within this range are considered to represent real values, as reported in another online 2D:4D study. ${ }^{22}$ Nevertheless, the 2D:4D ratios in our study have larger SD than those in an experimenter-measured finger length study. ${ }^{26}$ Our calculated 2D:4Ds therefore have relatively high levels of random error, with weakening of any relationships between 2D:4D and ALS. This can also be seen from our gender difference findings, which while significant and being close to those previously reported, ${ }^{27}$ had smaller effect sizes, with a reduction from $d$ values of 
$0.5-0.6$ in experimenter-measured studies ${ }^{26}$ to $0.25-0.35$ in our online study. This emphasises the need for large numbers of subjects in web-based 2D:4D studies.

Ideally, to be able to confidently exclude small betweengroup differences in 2D:4D, future studies in ALS would be of about 200 male and female ALS and control subjects (ie, 800 subjects in all). In addition to direct experimenter measurements of finger lengths, it would be useful to photograph the hands under consistent conditions (eg, image size, lighting, camera-finger distance) and use a computer-based measurement tool on the images to assess inter-rater and test-retest reliability. However, undertaking such a large study in a single clinical setting would be difficult. As a rule of thumb, any study that is insufficiently powered to detect the known gender differences in 2D:4D is unlikely to be able to detect biologically significant 2D:4D differences between disease and control groups.

Other limitations of our study are: (1) in order for respondents to remain anonymous they had to self-report their ALS status. We could not therefore classify their types of ALS using El Escorial criteria or more recently suggested classification methods. ${ }^{28}$ However, self-reporting of ALS status has previously been found to be $94 \%$ accurate when compared with physicians' responses. ${ }^{29}$ Indirect confirmation of an accurate ALS diagnosis is suggested by $88 \%$ of ALS respondents being able to choose their subtype of ALS via a multiple choice question (patients were encouraged to contact their physician if they were unsure of their subtype) and by $98 \%$ of ALS respondents completing the ALSFRS-R, which when completed online has been shown to compare well with clinical evaluation. (2) The results may be biased towards selecting people with early ALS, since people with advanced ALS are those who would be most likely to be unable to straighten their fingers for measurements. However, there is no evidence that the pathogenesis of ALS differs between the early and later stage of the disease, so this should not affect our conclusions. (3) Some people with ALS have cognitive deficits $^{30}$ and so may not have been able to measure their fingers accurately. However, it is unlikely that anybody with a clinically significant cognitive deficit would have been able to complete the extensive online questionnaire.

Our results do not corroborate the findings of a previous study that suggested the 2D:4D is lower in patients with ALS than in controls. ${ }^{18}$ This study had modest numbers of participants, with 33 ALS males $(70 \%)$ and 14 ALS females $(30 \%)$, compared with 20 control males $(32 \%)$ and 43 control females $(68 \%)$. The combination of an excess of males (with gender-related $2 \mathrm{D}<4 \mathrm{D}$ ) in the ALS group and an excess of females (with gender-related $2 \mathrm{D} \geq 4 \mathrm{D}$ ) in the control group could have led to a finding of a decreased mean 2D:4D in the ALS group, though raw results were not available to test this. The authors of this study acknowledge that insufficient numbers were available to look for 2D:4D gender differences. ${ }^{18}$ Our failure to replicate the results of this study are perhaps not surprising, given that it is androgen deficiency that affects motoneurons adversely, ${ }^{31}{ }^{32}$ so androgen excess during development (which causes a low 2D:4D) would not a priori be expected to be a risk factor for later motoneuron loss.

A lower 2D:4D ratio is found in people who have athletic ability. ${ }^{33}$ Based on the previous report of a lower 2D:4D in ALS patients, ${ }^{18}$ it was hypothesised that the suspected association of physical activity with ALS is because people with ALS have a prenatal disposition to both ALS and athleticism due to exposure to higher prenatal testosterone, rather than the physical activity itself being responsible for the increased ALS risk. ${ }^{18} 34$ Our finding of no lower 2D:4D in ALS makes this hypothesis unlikely. Moreover, the evidence for physical activity as a risk factor for ALS has recently been questioned. ${ }^{35}$ However, if further studies confirm this ALS-exercise link, other reasons for physical activity being associated with ALS need to be considered, such as those listed for football (soccer) players who have an increased risk of ALS, which include the use of toxic substances to improve performance, exposure to environmental toxins such as fertilisers or herbicides on playing fields or sport-related trauma. ${ }^{36}$ A further suggestion that has been put forward to explain an ALS-exercise link is that exercise increases transmission of circulating toxicants across the neuromuscular junction into motor axons, from where the toxicants travel to the motoneuron cell body by retrograde axonal transport and trigger the disease. ${ }^{37}$

Three future 2D:4D studies could be undertaken once more responses to international questionnaires such as ours are available. (1) A study using self-measured finger lengths has shown that the $2 \mathrm{D}: 4 \mathrm{D}$ varies with ethnicity ${ }^{38}$ (this would not have affected our results since our respondents were mostly of European origin). In addition, 2D:4Ds may be different within gene pools from people of the same ethnicity. ${ }^{39}$ Examination of ALS-related 2D:4D differences in various ethnic groups and between different nations will be possible once more respondents from other ethnic groups and countries provide relevant finger length data. (2) The 2D:4D appears to be lower in people born in late autumn, possibly because of the effects of melatonin on fetal testosterone. ${ }^{40}$ This time of season overlaps with the same period (late summer to early winter) in which ALS birth rates have been reported to be increased. ${ }^{41}$ A future study on season/ALS/2D:4D interactions will be possible once sufficient data on these variables are collected. (3) Between-nation gender differences in 2D:4D have been described ${ }^{42}$ so it would be of interest to gather sufficient numbers of ALS and control responses from different countries to be able to compare 2D:4Ds between these countries.

In conclusion, our online case-control study found no differences between the 2D:4Ds of ALS and non-ALS respondents. The study does not therefore support the hypothesis that exposure to prenatal testosterone is a risk factor for ALS. If physical activity is confirmed to be a risk factor for ALS, reasons other than a low 2D:4D need to be sought to explain this association. Future studies 
of ethnic, time-of-birth and multination subgroups may detect more nuanced relationships between ALS and 2D:4D when data from larger numbers of ALS and non-ALS respondents become available.

Acknowledgements We thank all respondents who took the time to complete the detailed ALS Quest questionnaire, and volunteers who helped translate the questionnaire into different languages. Respondents were alerted to the availability of the questionnaire by many worldwide national and state-based ALS associations, the International Alliance of ALS/MND Associations, the USA CDC National ALS Registry, the Canadian Neuromuscular Disease Registry and community clubs such as those run by Rotary, Lions, the Returned and Services League and the Men's Shed Association. Lucidchart software was used to create the flow diagram.

Contributors JAPK and RP planned the project, acquired and analysed the data, wrote the manuscript (JAPK wrote the first draft), approved the final version submitted and are accountable for all aspects of the work.

Funding Supported by the Aimee Stacy Memorial and Ignacy Burnett Motor Neuron Disease bequests.

Competing interests The study was partially funded by the Aimee Stacy Memorial Bequest and the Ignacy Burnett Motor Neuron Disease Bequest, both managed by the University of Sydney.

Patient consent Participants consented to answering the online questionnaire by following these instructions: "Please click on the I Consent button below to indicate your consent to enter data into the questionnaire. By clicking this button, I: 1. Acknowledge that I have read the Information for Participants above and agree to participate in this research. 2. Understand that I will not be asked for any personal information that could identify me, so the study is anonymous and strictly confidential. 3. Freely choose to participate in the study and understand that I can withdraw my questionnaire answers at any time until I click the Submit button at the end of the questionnaire."

Ethics approval The Human Ethics Committee of the Sydney Local Health District.

Provenance and peer review Not commissioned; externally peer reviewed.

Data sharing statement No additional unpublished data from the study (apart from the two supplementary files) are available.

Open Access This is an Open Access article distributed in accordance with the Creative Commons Attribution Non Commercial (CC BY-NC 4.0) license, which permits others to distribute, remix, adapt, build upon this work non-commercially, and license their derivative works on different terms, provided the original work is properly cited and the use is non-commercial. See: http://creativecommons.org/ licenses/by-nc/4.0/

C Article author(s) (or their employer(s) unless otherwise stated in the text of the article) 2017. All rights reserved. No commercial use is permitted unless otherwise expressly granted.

\section{REFERENCES}

1. Baker F. Anthropological notes on the human hand. Am Anthropol 1888;A1:51-76.

2. Manning JT, Scutt D, Wilson J, et al. The ratio of 2 nd to 4 th digit length: a predictor of sperm numbers and concentrations of testosterone, luteinizing hormone and oestrogen. Hum Reprod 1998;13:3000-4.

3. Manning JT, Taylor RP. Second to fourth digit ratio and male ability in sport: implications for sexual selection in humans. Evol Hum Behav 2001;22:61-9.

4. Manning JT, Bundred PE. The ratio of 2 nd to 4th digit length: a new predictor of disease predisposition? Med Hypotheses 2000;54:855-7.

5. Lutchmaya S, Baron-Cohen S, Raggatt P, et al. 2nd to 4th digit ratios, fetal testosterone and estradiol. Early Hum Dev 2004;77:23-8.

6. Zheng Z, Cohn MJ. Developmental basis of sexually dimorphic digit ratios. Proc Natl Acad Sci U S A 2011;108:16289-94.

7. Manning JT. Resolving the role of prenatal sex steroids in the development of digit ratio. Proc Natl Acad Sci U S A 2011;108:16143-4.

8. Hönekopp J, T Manning J, Müller C. Digit ratio (2D:4D) and physical fitness in males and females: Evidence for effects of prenatal androgens on sexually selected traits. Horm Behav 2006;49:545-9.
9. Hull MJ, Schranz NK, Manning JT, et al. Relationships between digit ratio (2D:4D) and female competitive rowing performance. Am J Hum Biol 2015;27:157-63.

10. Ribeiro E, Neave N, Morais RN, et al. Digit ratio (2D:4D), testosterone, cortisol, aggression, personality and hand-grip strength: Evidence for prenatal effects on strength. Early Hum Dev 2016;100:21-5.

11. Coates JM, Gurnell M, Rustichini A. Second-to-fourth digit ratio predicts success among high-frequency financial traders. Proc Natl Acad Sci U S A 2009;106:623-8.

12. Williams TJ, Pepitone ME, Christensen SE, et al. Finger-length ratios and sexual orientation. Nature 2000;404:455-6.

13. Muller DC, Baglietto L, Manning JT, et al. Second to fourth digit ratio (2D:4D), breast cancer risk factors, and breast cancer risk: a prospective cohort study. Br J Cancer 2012;107:1631-6.

14. Muller DC, Giles GG, Manning JT, et al. Second to fourth digit ratio (2D:4D) and prostate cancer risk in the Melbourne Collaborative Cohort Study. Br J Cancer 2011;105:438-40.

15. Vladeanu M, Giuffrida O, Bourne VJ. Prenatal sex hormone exposure and risk of Alzheimer disease: a pilot study using the 2D:4D digit length ratio. Cogn Behav Neurol 2014;27:102-6.

16. Bove R, Malik MT, Diaz-Cruz C, et al. The 2D:4D ratio, a proxy for prenatal androgen levels, differs in men with and without MS. Neurology 2015;85:1209-13.

17. Kiernan MC, Vucic S, Cheah BC, et al. Amyotrophic lateral sclerosis. Lancet 2011;377:942-55.

18. Vivekananda U, Manjalay ZR, Ganesalingam J, et al. Low indexto-ring finger length ratio in sporadic ALS supports prenatally defined motor neuronal vulnerability. J Neurol Neurosurg Psychiatry 2011;82:635-7

19. Parkin Kullmann JA, Hayes S, Wang MX, et al. Designing an Internationally Accessible Web-Based Questionnaire to Discover Risk Factors for Amyotrophic Lateral Sclerosis: A Case-Control Study. JMIR Res Protoc 2015;4:e96.

20. Cedarbaum JM, Stambler N, Malta E, et al. The ALSFRS-R: a revised ALS functional rating scale that incorporates assessments of respiratory function. BDNF ALS Study Group (Phase III). J Neurol Sci 1999;169:13-21.

21. Faul F, Erdfelder E, Lang AG, et al. G*Power 3: a flexible statistical power analysis program for the social, behavioral, and biomedical sciences. Behav Res Methods 2007;39:175-91.

22. Collaer ML, Reimers S, Manning JT. Visuospatial performance on an internet line judgment task and potential hormonal markers: sex, sexual orientation, and 2D:4D. Arch Sex Behav 2007;36:177-92.

23. Gosling SD, Vazire S, Srivastava S, et al. Should we trust web-based studies? A comparative analysis of six preconceptions about internet questionnaires. Am Psychol 2004;59:93-104.

24. Reimers S. The BBC internet study: general methodology. Arch Sex Behav 2007;36:147-61.

25. Caswell N, Manning JT. A comparison of finger 2D:4D by selfreport direct measurement and experimenter measurement from photocopy: methodological issues. Arch Sex Behav 2009;38:143-8.

26. Voracek M, Manning JT, Dressler SG. Repeatability and interobserver error of digit ratio (2D:4D) measurements made by experts. Am J Hum Biol 2007;19:142-6.

27. Manning JT. Digit ratio: a pointer to fertility, behavior and health. New Brunswick, NJ: Rutgers University Press, 2002.

28. Al-Chalabi A, Hardiman O, Kiernan MC, et al. Amyotrophic lateral sclerosis: moving towards a new classification system. Lancet Neurol 2016;15:1182-94.

29. Malek AM, Stickler DE, Antao VC, et al. The National ALS Registry: a recruitment tool for research. Muscle Nerve 2014;50:830-4.

30. Phukan J, Pender NP, Hardiman O. Cognitive impairment in amyotrophic lateral sclerosis. Lancet Neurol 2007;6:994-1003.

31. Cary GA, La Spada AR. Androgen receptor function in motor neuron survival and degeneration. Phys Med Rehabil Clin N Am 2008;19:479-94.

32. Yoo YE, Ko CP. Dihydrotestosterone ameliorates degeneration in muscle, axons and motoneurons and improves motor function in amyotrophic lateral sclerosis model mice. PLoS One 2012;7:e37258

33. Manning JT, Morris L, Caswell N. Endurance running and digit ratio (2D:4D): implications for fetal testosterone effects on running speed and vascular health. Am J Hum Biol 2007;19:416-21.

34. Wicks P. Hypothesis: higher prenatal testosterone predisposes ALS patients to improved athletic performance and manual professions. Amyotroph Lateral Scler 2012;13:251-3.

35. Lacorte E, Ferrigno L, Leoncini E, et al. Physical activity, and physical activity related to sports, leisure and occupational activity as risk factors for ALS: A systematic review. Neurosci Biobehav Rev 2016;66:61-79. 
36. Chiò A, Benzi G, Dossena M, et al. Severely increased risk of amyotrophic lateral sclerosis among Italian professional football players. Brain 2005;128:472-6.

37. Pamphlett R, Kum Jew S. Uptake of inorganic mercury by human locus ceruleus and corticomotor neurons: implications for amyotrophic lateral sclerosis. Acta Neuropathol Commun 2013;1:13.

38. Manning JT, Churchill AJ, Peters M. The effects of sex, ethnicity, and sexual orientation on self-measured digit ratio (2D:4D). Arch Sex Behav 2007;36:223-33.
39. Loehlin JC, McFadden D, Medland SE, et al. Population differences in finger-length ratios: ethnicity or latitude? Arch Sex Behav 2006;35:739-42.

40. Szwed A, Kosinska M, Manning JT. Digit ratio (2D:4D) and month of birth: A link to the solstitial-melatonin-testosterone effect. Early Hum Dev 2017;104:23-6.

41. Pamphlett R, Fang F. Season and weather patterns at time of birth in amyotrophic lateral sclerosis. Amyotroph Lateral Scler 2012;13:459-64.

42. Manning JT, Fink B, Trivers R. Digit ratio (2D:4D) and gender inequalities across nations. Evol Psychol 2014;12:757-68. 